\title{
Doping-Tunable Ferrimagnetic Phase with Large Linear Magnetoelectric Effect in a Polar Magnet $\mathrm{Fe}_{2} \mathrm{Mo}_{3} \mathrm{O}_{8}$
}

\author{
T. Kurumaji, ${ }^{1, *}$ S. Ishiwata, ${ }^{1}$ and Y. Tokura ${ }^{1,2}$ \\ ${ }^{1}$ Department of Applied Physics and Quantum Phase Electronics Center (QPEC), \\ University of Tokyo, Tokyo 113-8656, Japan \\ ${ }^{2}$ RIKEN Center for Emergent Matter Science (CEMS), Wako 351-0198, Japan \\ (Received 21 February 2015; revised manuscript received 11 August 2015; published 15 September 2015)

\begin{abstract}
The magnetoelectric (ME) effect, i.e., cross control of magnetization (electric polarization) by an external electric (magnetic) field, may introduce a new design principle for novel spin devices. To enhance the ME signal, control of a phase competition has recently been revealed as a promising approach. Here, we report the successful chemical-doping control of the distinct ME phases in a polar magnet $\mathrm{Fe}_{2} \mathrm{Mo}_{3} \mathrm{O}_{8}$, in which an antiferromagnetic state is competing with a ferrimagnetic state. We demonstrate that $\mathrm{Zn}$ doping stabilizes the metamagnetic state to realize the spontaneous ferrimagnetic state and varies the ME coefficients from large negative to large positive values; for instance, the diagonal component of the ME by doping $\mathrm{Zn}$ from $12.5 \%$ to $50 \%$. This remarkable doping control of the ME property originates from coexisting distinct ME mechanisms, which are selectively tunable by substituting one of the two distinct magnetic sites in the unit cell with nonmagnetic $\mathrm{Zn}$.
\end{abstract} \\ coefficients under the magnetic field perpendicular to the polar axis varies from $-142 \mathrm{ps} / \mathrm{m}$ to $107 \mathrm{ps} / \mathrm{m}$
}

DOI: 10.1103/PhysRevX.5.031034

\section{INTRODUCTION}

Since the discovery of the magnetoelectric (ME) effect in chromate [1,2], the research on the ME properties has developed steadily and attracted much attention because of its potential application in novel electronic devices [3-5]. This field of research has recently experienced a revival [6] promoted by the engineered ME effect in composite systems and the continual search for new multiferroics, where plural (dielectric and magnetic) orders coexist [7-9]. In the latter field, recent advances include, for example, the nonlinear magnetic-field $(H)$ induced ferroelectric-polarization switch, thin-film growth techniques to stabilize large polarization coupled with magnetism, and the room-temperature multiferroic property in perovskite oxides [10-12].

Along with continuing efforts to realize the roomtemperature operation and colossalization of the ME signal, improvement of designability of ME materials and properties is an important step towards the application. One of the promising approaches is the chemical modification of multiferroic/ME materials, for example, towards the stabilization of the multiferroic phase in a higher temperature

\footnotetext{
*Corresponding author. kurumaji@cmr.t.u-tokyo.ac.jp

Published by the American Physical Society under the terms of the Creative Commons Attribution 3.0 License. Further distribution of this work must maintain attribution to the author(s) and the published article's title, journal citation, and DOI.
}

Subject Areas: Condensed Matter Physics, Magnetism, Strongly Correlated Materials range; a good example of this in the field of ferroelectrics is the solid solution (mixed-crystal engineering) of Pb-based perovskite oxides [12]. For further improvement of controllability of the ME effect, in this study, we focus on the chemical-doping effect on the ME property in the magnetically frustrated system, where plural distinct magnetic phases are competing on a subtle balance of free energy.

A possible advantage of utilizing the frustrated system is that the competing magnetic interactions can be easily perturbed by the chemical-doping procedure on the specific magnetic site, which may enable the sensitive control of competing multiferroic phases and/or the ME property, as exemplified by the case of Ga- and Al-doping-induced stabilization of the ferroelectric phase in the triangularlattice antiferromagnet $\mathrm{CuFeO}_{2}$ [13], for instance, and $\mathrm{Zn}$-doping-induced modulation of the multiferroic phase in $\mathrm{MnWO}_{4}$ [14]. One of the problems of this strategy is that the ion-substitution may easily dilute the magnetism and also the magnitude of the ME signal. Here, we report one solution to overcome such a difficulty; we can chemically control the competition among different magnetic phases with respective ME properties and the variation of the magnitude, and even the sign of the linear $\mathrm{ME}$ effect without losing its hugeness in a $\mathrm{ME}$ material $\mathrm{Fe}_{2} \mathrm{Mo}_{3} \mathrm{O}_{8}$ with crystallographic polarity.

The family of $M_{2} \mathrm{Mo}_{3} \mathrm{O}_{8}$ ( $M: 3 d$ transition metal) belongs to a pyroelectric space group $P 6_{3} m c$ [15], which allows the crystallographic polarity along the $c$ axis. The crystal structure can be viewed as the stacking of $M_{2}$ layers 
(a)

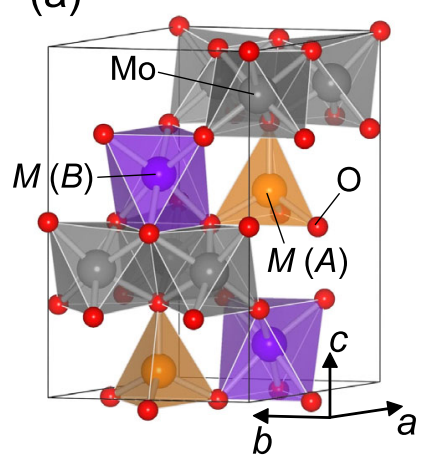

(b) antiferromagnetic

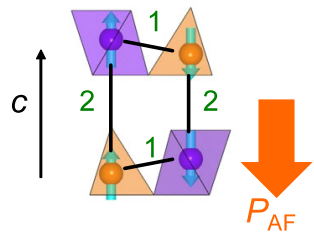

(c) ferrimagnetic

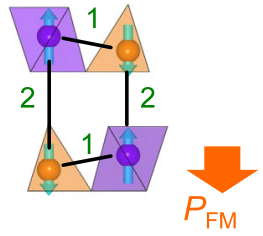

FIG. 1. (a) A unit cell of $M_{2} \mathrm{Mo}_{3} \mathrm{O}_{8}(M: \mathrm{Fe})$. (b, c) Schematic illustrations of antiferromagnetic and ferrimagnetic orders, respectively. Spin-induced $P\left(P_{\mathrm{AF}}\right.$ and $\left.P_{\mathrm{FM}}\right)$ are also indicated. Although the sign of $P$ with respect to the $c$ axis has not been determined, it is provisionally defined in this work. The numbers 1 and 2 are the indices for bonds of magnetic interaction.

and $\mathrm{Mo}_{3}$ layers along the $c$ axis, alternatively intervening between the closest packing of oxygen. The $M_{2}$ layer consists of two kinds of sites, $A$ and $B$ [Fig. 1(a)], which are in tetrahedral and octahedral coordinations of oxygen, respectively. Crystallographic polarity manifests itself as the polar arrangement of $A_{4}$ tetrahedra. The magnetism of the system is dominated by unpaired $3 d$ electrons in $M^{2+}$ ions at both $A$ and $B$ sites, while $\mathrm{Mo}^{4+}$ ions form spin-singlet trimers [16]. Previous magnetization and powder neutron diffraction studies [17-19] for $M=\mathrm{Fe}$ unraveled its antiferromagnetic ground state [Fig. 1(b)] with $T_{\mathrm{N}}=60 \mathrm{~K}$.

An interesting feature for the presently investigated material $\mathrm{Fe}_{2} \mathrm{Mo}_{3} \mathrm{O}_{8}$ is $\mathrm{Zn}$-doping tunability between distinct magnetic ground states, suggesting the frustration among the magnetic interactions. The powder neutron diffraction study of $(\mathrm{Fe}, \mathrm{Zn})_{2} \mathrm{Mo}_{3} \mathrm{O}_{8}$ in Ref. [18] reported that the ferrimagnetic ground state [Fig. 1(c)] is stabilized for samples with $\mathrm{Zn}$ substituting more than $15 \%$ of $\mathrm{Fe}$. Such tunability was understood by the selective dilution of competing magnetic interactions from the fact that $\mathrm{Zn}$ atoms prefer to occupy the tetrahedrally coordinated $A$ sites [19].

Here, we systematically investigate magnetic and dielectric properties of $\mathrm{Fe}_{2} \mathrm{Mo}_{3} \mathrm{O}_{8}$ and the $\mathrm{Zn}$-doping effect on them. In $\mathrm{Fe}_{2} \mathrm{Mo}_{3} \mathrm{O}_{8}$, we find that the applied magnetic field switches the antiferromagnetic order into the ferrimagnetic order, and we demonstrate that the latter phase shows a large linear ME effect. Upon Zn doping, we observe the expansion of the hysteretic region between these two magnetic phases; these phase-competition features controlled by both magnetic field and chemical doping are reminiscent of the features in the colossal magnetoresistive manganites [20]. We further observe, in the stabilized ferrimagnetic phase with spontaneous magnetization, the changes of the ME coefficients from large negative to large positive values. We suggest that the wide-range control of the linear ME effect originates from the coexistence of competing ME mechanisms and their selective tunability by Zn doping.

\section{SAMPLE PREPARATION AND CHARACTERIZATION}

The single crystal of $\mathrm{Fe}_{2} \mathrm{Mo}_{3} \mathrm{O}_{8}$ was grown by chemical vapor transport [21,22]. The single crystals of $\left(\mathrm{Fe}_{1-y} \mathrm{Zn}_{y}\right)_{2} \mathrm{Mo}_{3} \mathrm{O}_{8}(y=0.05 \sim 0.5)$ were grown from the mixture of $\mathrm{MoO}_{2}+\mathrm{Fe}+\mathrm{Fe}_{2} \mathrm{O}_{3}+\mathrm{ZnO}$ with the molar ratio $\mathrm{Mo}^{4+}: \mathrm{Fe}^{2+}: \mathrm{Zn}^{2+}=1.5: 1-y: y$. The content of $y$ in the samples was checked by the transition temperature compared with that reported in Ref. [18]. Magnetization was measured with a SQUID magnetometer (MPMS; Quantum Design) and the AC/DC Magnetometry System (PPMS; Quantum Design). For dielectric measurements, silver paste was painted on the parallel end surfaces of the specimen as the electrodes. Electric polarization $P$ was deduced by time integration of the polarization current measured with constant rates of $T$ sweep $(10 \mathrm{~K} / \mathrm{min})$ and $H$ sweep $(80 \mathrm{Oe} / \mathrm{sec})$ using an electrometer (Keithley Model 6517A). The plus direction of $P$ along the $c$ axis is commonly determined for all samples by the sign of pyroelectricity in the paramagnetic phase.

\section{RESULTS}

The temperature $(T)$ dependence of magnetic susceptibility $(M / H)$ parallel to the $c$ axis was measured under $\mu_{0} H=0.5 \mathrm{~T}$ [Fig. 2(a)]. The sharp peak in $M / H$ suggests the antiferromagnetic ordering [Fig. 1(b)] at $T_{\mathrm{N}}=60 \mathrm{~K}$ in accordance with the previous neutron diffraction study [18]. What is unraveled by the present study is that the magnetic transition is accompanied by modulation of the electric polarization $(P)$ along the polar $c$ axis, as shown by the $T$ dependence of $P$ at $\mu_{0} H=0$ T [Fig. 2(b)]. In the paramagnetic phase for $T>T_{\mathrm{N}}, P$ gradually increases as it is cooled from $140 \mathrm{~K}$ because of the pyroelectric effect. A steep drop in $P$ at $T_{\mathrm{N}}$ suggests that the magnetic order induces additional $P$ to the crystallographic $P$. $P$ starts to decrease from $T$ higher than $T_{\mathrm{N}}$ by $20 \mathrm{~K}$, possibly because of the formation of short-range order above $T_{\mathrm{N}}$.

The $T$ dependence of $M / H$ and $P$ dramatically changes with an increase of $H(\| c)$. The sharp transition peak of $M / H$ becomes broad, followed by a first-order phase transition at lower $T$ indicated by closed triangles in Fig. 2(a), suggesting successive magnetic phase transitions under $H$. These sequential transitions are also evident in the $T$ dependence of $P$ under $H$ up to $14 \mathrm{~T}$; $P$ shows a nonmonotonous and continuous change until it shows a sudden drop when $T$ reaches the first-order phase transition point indicated by open triangles in Fig. 2(b). To elucidate 

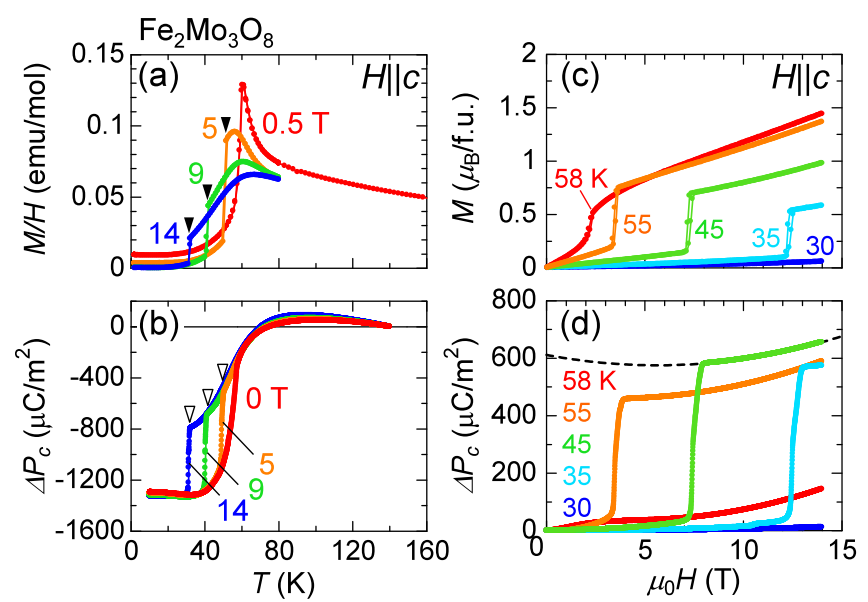

FIG. 2. (a, b) $T$ dependence of (a) $M / H$ and (b) change of $P$ $(\Delta P)$ under various magnitudes of $H$ along the $c$ axis. (c, d) Field dependence of $M$ and $\Delta P$ at various $T$. The dashed curve is the fit to the $P-H$ curve for $H>H_{\text {cr }}$ at $45 \mathrm{~K}$ with the function $P=P_{0}+\alpha_{3} H+\beta H^{2}$, where $P_{0}, \alpha_{3}$, and $\beta$ are constants. Zero points of $\Delta P$ are taken at (b) $T=140 \mathrm{~K}$ and (d) $\mu_{0} H=0 \mathrm{~T}$.

the magnetic phases of this compound, we show $H$ dependence of $M$ and $P$ under $H \| c$ measured at various $T$ in Figs. 2(c) and 2(d). Each $M-H$ curve shows a clear stepwise structure indicating a metamagnetic transition, while a jump of $P$ can be clearly seen in the $H$ scan. The transition of the magnetic ground state from the antiferromagnetic order [Fig. 1(b)] to the ferrimagnetic one [Fig. 1(c)] by $\mathrm{Zn}$ doping was reported in a previous study [18]. Thus, it is likely that these two magnetic phases are competing with each other even in the parent compound $\mathrm{Fe}_{2} \mathrm{Mo}_{3} \mathrm{O}_{8}$.

Figure 3(a) shows the $H$ - $T$ phase diagram determined by the magnetic and dielectric anomalies in $T$ and $H$ scans of $M$ and $P$ under $H \| c$. The ferrimagnetic phase (denoted as "FM" in the figure) is identified under $H \| c$. A strong correlation between magnetism and electricity of this system is indicated by a good coincidence of the magnetic

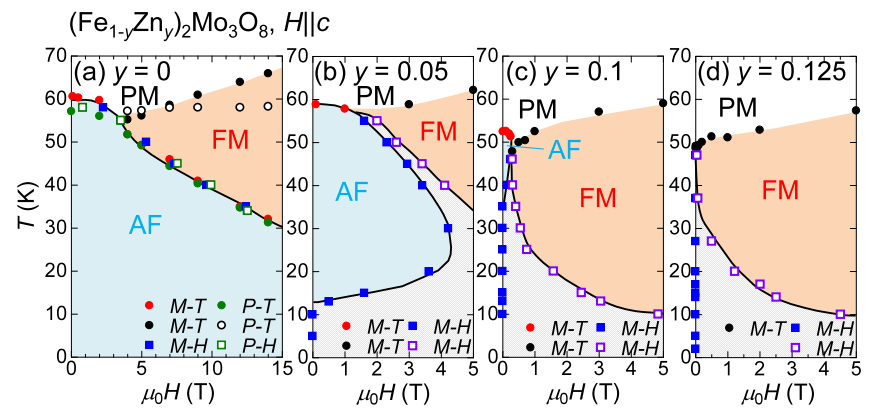

FIG. 3. (a-d) The $H-T$ phase diagrams for $\left(\mathrm{Fe}_{1-y} \mathrm{Zn}_{y}\right)_{2} \mathrm{Mo}_{3} \mathrm{O}_{8}$ with various values of $y$ under $H \| c$, as determined by $M$ and $P$ measurements. PM, AF, and FM indicate the paramagnetic, antiferromagnetic, and ferrimagnetic phases, respectively. Shaded areas indicate hysteretic regions. and dielectric anomalies. A slight discrepancy in the phase boundary between PM and FM (closed and open black circles) is due to the absence of well-defined anomalies in estimating the transition points, which were determined by the maxima of $T$ derivatives of $P$ and $M$, respectively.

We note that the ferrimagnetic phase is characterized by a linear ME effect. The ME tensor $\hat{\alpha}$ for the ferrimagnetic structure belonging to the magnetic point group $6 m^{\prime} m^{\prime}$ is given by symmetry as [23]

$$
\hat{\alpha}=\left(\begin{array}{ccc}
\alpha_{1} & 0 & 0 \\
0 & \alpha_{1} & 0 \\
0 & 0 & \alpha_{3}
\end{array}\right) .
$$

In Fig. 2(d), while $P$ is quadratic to $H$ for $H<H_{\text {cr }}\left(H_{\mathrm{cr}}\right.$ : critical field for the metamagnetic transition), for $H>H_{\text {cr }}$ it is well fitted with the formula $\Delta P=P_{0}+\alpha_{3} H+\beta H^{2}$, including the $H$-linear term. $P_{0}$ is the genuine $(H=0)$ difference of $P$ between the antiferromagnetic and ferrimagnetic structures, and $\beta$ corresponds to the second-order ME effect. We exemplify one of the fits as the dashed curve in Fig. 2(d). We obtain $\alpha_{3}=-16.2 \mathrm{ps} / \mathrm{m}$ as well as $P_{0}=611 \mu \mathrm{C} / \mathrm{m}^{2}$ and $\beta=1.81 \times 10^{-28} \mathrm{~s} / \mathrm{A}$. This result is consistent with the fact that the magnetic point group for the antiferromagnetic order $\left(6^{\prime} \mathrm{mm}^{\prime}\right)$ allows only the second-order ME effect, while the ferrimagnetic order $\left(6 m^{\prime} m^{\prime}\right)$ allows the linear ME effect under $H \| c$.

To clarify the link between the metamagnetic phase identified in $\mathrm{Fe}_{2} \mathrm{Mo}_{3} \mathrm{O}_{8}$ under $H \| c$ and the ferrimagnetic order stabilized by $\mathrm{Zn}$ doping, we show magnetization of $\left(\mathrm{Fe}_{1-y} \mathrm{Zn}_{y}\right)_{2} \mathrm{Mo}_{3} \mathrm{O}_{8}$ with various $y$ in Figs. 4(a)-4(e). Figures 4(a) and 4(b) show the $T$ dependence of $M$ along the $c$ axis under $\mu_{0} H=0.1 \mathrm{~T}$. For $y \leq 0.1$, the magnetization peak upon the antiferromagnetic transition becomes sharper by increasing $y$, suggesting incipient ferrimagnetism. Clear ferromagnetic behaviors were observed for

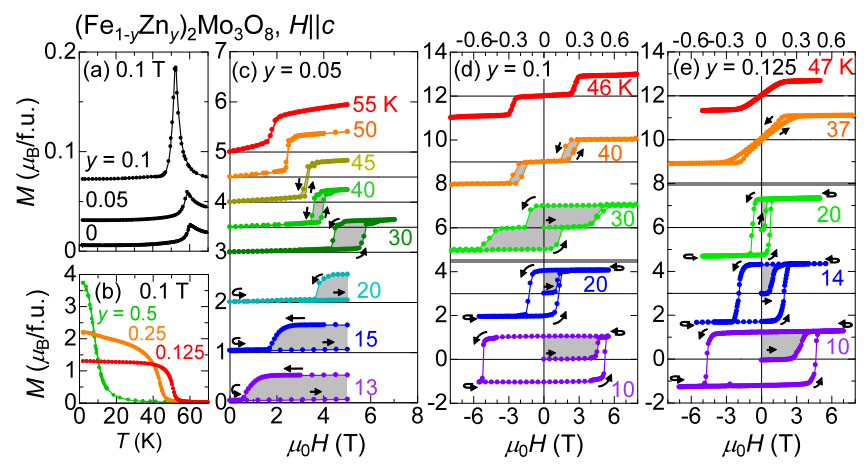

FIG. 4. (a, b) $T$ and (c-e) $H$ dependence of $M$ for $\left(\mathrm{Fe}_{1-y} \mathrm{Zn}_{y}\right)_{2} \mathrm{Mo}_{3} \mathrm{O}_{8}$ with various values of $y$ under $H \| c$. (c-e) Each scan starts after cooling to the target $T$ without a field. The horizontal scale of $\mu_{0} H$ for data above the gray horizontal bars in (d) and (e) is appended at the top of each panel. Data for (a) and (c-e) are shifted for clarity. 
$y \geq 0.125$ in accordance with the previous study on the polycrystalline samples [24].

$\mathrm{Zn}$-induced stabilization of the metamagnetic phase transition is clear from the $M-H$ scan for $y=0.05$ [Fig. 4(c)], which shows a dramatic reduction of $H_{\mathrm{cr}}$. The ferrimagnetic phase stabilized by $\mathrm{Zn}$ doping is verified even more clearly for $y=0.1$ and 0.125 [Figs. 4(d) and 4(e)]. Note that hysteresis [shaded area in Figs. 4(c)-4(e)] shows up as lowering $T$. The feature is reminiscent of the first-order phase transition induced by $H$ in colossal magnetoresistance compounds [20]. Expansion of the hysteretic region is qualitatively assigned to the suppressed thermal fluctuation that preserves the metastable state as the supercooled state, even at $H$ parting from the true $H_{\text {cr }}$ [25]. Based on these results, the $H-T$ phase diagrams for various $y$ are displayed in Figs. 3(b)-3(d). It is evident that the area of the antiferromagnetic phase shrinks as $y$ increases, and for $y=0.125$, it only appears as a metastable state. Thus, we conclude that the metamagnetic phase transition for $\mathrm{Fe}_{2} \mathrm{Mo}_{3} \mathrm{O}_{8}$ generates the ferrimagnetic order [Fig. 1(c)] and that Zn-doping controls the competition between the antiferromagnetic and ferrimagnetic orders.

As observed in the metamagnetic phase in $\mathrm{Fe}_{2} \mathrm{Mo}_{3} \mathrm{O}_{8}$, the $\mathrm{Zn}$-induced ferrimagnetic state shows the corresponding linear ME effect. According to Eq. (1), the magnetic symmetry only allows the diagonal components of the ME tensor $\hat{\alpha}$ for the ferrimagnetic structure [23]. We investigate $\alpha_{3}\left(\Delta P_{c}=\alpha_{3} H_{c}\right)$ at first by measuring $P$ along the $c$ axis under $H \| c$ for $y=0.125,0.25$, and 0.5 [Fig. 5(b)]. In these measurements, the sample was first cooled with $H=0$; then at the lowest $T$, we scanned $H$ from $0 \rightarrow+H,+H \rightarrow-H$, and $-H \rightarrow+H$. During the scans $+H \rightarrow-H \rightarrow+H, P$ for each $y$ shows a butterflylike hysteretic curve. The $P-H$ hysteretic region is in accordance with the $M-H$ hysteretic region [Fig. 5(a)]. This is typical of ferromagnetic linear ME materials such as Ni-boracite [26] and $\mathrm{GaFeO}_{3}$ [27], in which the sign of the linear ME coefficient is reversed upon the $H$-induced ferromagnetic domain reversal. The linear fittings to $P$ during the scans $+H \rightarrow-H$ are shown by black-dashed lines in Fig. 5(b). To eliminate the multidomain contribution, we performed the fitting on $5 \mathrm{~T}<\mu_{0} H<14 \mathrm{~T}$ for $y=0.125$ and 0.25 , and $0 \mathrm{~T}<\mu_{0} H<9 \mathrm{~T}$ for $y=0.5$, respectively. These fittings extrapolate to $0 \mathrm{~T}$ with only a slight deviation, signaling the linear ME response intrinsic to the ferrimagnetic phase. For $y=0.125$, we observe a large difference in $P$ at $\mu_{0} H=0$ T between the initial scan (black curve) and the field-trained state (red curve). This suggests that the antiferromagnetic phase remains, for $y=0.125$ after the zero-field cooling, as the metastable state, while the ferrimagnetic ground state is realized only after the field scan. This discrepancy between the trained and untrained $P$ is observed to be smaller upon $\mathrm{Zn}$ doping (about $10 \mu \mathrm{C} / \mathrm{m}^{2}$ for $y=0.25$ and about $0 \mu \mathrm{C} / \mathrm{m}^{2}$ for

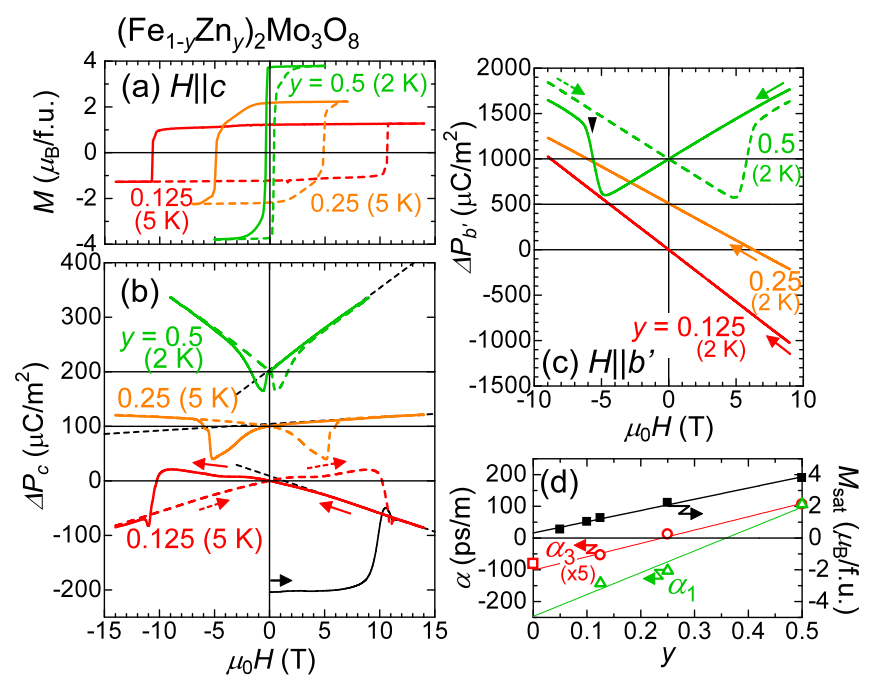

FIG. 5. (a-c) $H$ dependence of (a) $M$, (b) $\Delta P$ along the $c$ axis under $H \| c$, and (c) $\Delta P$ along the $b^{\prime}$ axis under $H \| b^{\prime}$. The $b^{\prime}$ axis is the axis intersecting at a right angle with one of the $a$ axes and the $c$ axis. The colored solid and dashed curves for each $y$ indicate the scans for $+H \rightarrow-H$ and $-H \rightarrow+H$, respectively. The black solid curve for $y=0.125$ in (b) is the scan from $\mu_{0} H=0 \mathrm{~T}$ to $14 \mathrm{~T}$ after the cooling without a field. Data of $\Delta P$ for each $y$ are shifted for clarity. Black dashed lines are linear fits to $P-H$ curves. (d) $y$ dependence of $\mathrm{ME}$ coefficients $\alpha_{1}$ and $\alpha_{3}$ measured at $2 \mathrm{~K}$, and saturated magnetization estimated from data in Figs. 4(c)-4(e) and Fig. 5(a). Solid lines are linear fits. The open square indicates the value of $\alpha_{3}$ for $\mathrm{Fe}_{2} \mathrm{Mo}_{3} \mathrm{O}_{8}$ at $45 \mathrm{~K}$, obtained from the data in Fig. 2(d).

$y=0.5$, not shown), indicating the gradual disappearance of the supercooled antiferromagnetic state.

We next investigate $\alpha_{1}\left(\Delta P_{b^{\prime}}=\alpha_{1} H_{b^{\prime}}\right)$ by measuring $H$ dependence of $P$ along the $b^{\prime}$ axis under $H \| b^{\prime}$ for $\left(\mathrm{Fe}_{1-y} \mathrm{Zn}_{y}\right)_{2} \mathrm{Mo}_{3} \mathrm{O}_{8}$ [Fig. 5(c)]. Prior to the measurements, the sample was slightly misaligned from $H \| b^{\prime}$ by about $1^{\circ}$ towards the $c$ axis and cooled to $2 \mathrm{~K}$ with $\mu_{0} H=9 \mathrm{~T}$, in order to fully polarize the ferromagnetic domain. The field was then scanned from $+9 \mathrm{~T}$ to $-9 \mathrm{~T}$. For $y=0.5$, clear $H$-linear dependence of $P$ is observed in the $+H$ region, while $P$ shows nonmonotonous change in the $-H$ region, indicated by a closed triangle. This corresponds to the ferromagnetic-domain flop induced by the $c$ component of $H$. For both $y=0.25$ and $y=0.125$, the $H$-linear $P$ in the whole field range ensures the $\alpha_{1}$ component of the ME tensor at $2 \mathrm{~K}$. To further verify the consistency of the above $\alpha_{1}$ with a $+c$-polarized ferromagnetic domain, we measured the $H$ dependence of $P_{b^{\prime}}$ under $H$ inclined from the $b^{\prime}$ axis towards the $+c$ axis by $60^{\circ}$, and we deduced the consistent value of $\alpha_{1}$ from $P_{b^{\prime}}=\alpha_{1} H \cos 60^{\circ}$.

We plot the $y$ dependence of $\alpha_{1}$ and $\alpha_{3}$ in Fig. 5(d), as estimated from $P-H$ measurements at $2 \mathrm{~K}$. We define the signs of $\alpha_{1}$ and $\alpha_{3}$ for each $y$ when the ferromagnetic polarization is along the $+c$ axis. Note that $\left(\mathrm{Fe}_{1-y} \mathrm{Zn}_{y}\right)_{2} \mathrm{Mo}_{3} \mathrm{O}_{8}$ of $y=0.5$ and $y=0.125$ show the 
largest values for $\alpha_{3}(22 \mathrm{ps} / \mathrm{m})$ and $\left|\alpha_{1}\right|(142 \mathrm{ps} / \mathrm{m})$, which are larger than those of typical linear $\mathrm{ME}$ compounds, including $\mathrm{Cr}_{2} \mathrm{O}_{3}(0.1 \sim 1 \mathrm{ps} / \mathrm{m})$ [3] and comparable with that of several well-known magnetoelectrics, including magnetic $\mathrm{Fe}$ ions such as $\mathrm{GaFeO}_{3}(21 \mathrm{ps} / \mathrm{m})$ [27,28] and $\mathrm{Fe}_{3} \mathrm{O}_{4}(50 \mathrm{ps} / \mathrm{m})$ [29-31], although other systems such as $\mathrm{TbPO}_{4}(300 \mathrm{ps} / \mathrm{m})$ [32], with a highly anisotropic rare-earth magnetic moment, and the composite systems such as $\mathrm{FeRh} / \mathrm{BaTiO}_{3}\left(1.6 \times 10^{-5} \mathrm{~s} / \mathrm{m}\right)$ [33] have been reported to show much larger ME coefficients. We also note that the observed linear ME effect shows significant $y$ dependence; both $\alpha_{1}$ and $\alpha_{3}$ change their signs as $y$ varies. Intuitively, doping nonmagnetic ions dilutes the magnetic interaction and would reduce the resultant ME response. Nevertheless, $\mathrm{Zn}$ doping is found to change $\alpha$ from a large negative to a large positive value.

Both $\alpha_{1}$ and $\alpha_{3}$ show $y$-linear dependence as in Fig. 5(d), which implies relevant correlation with the site occupancy of $\mathrm{Zn}$ for the $A$ site. The content of $\mathrm{Zn}$ at the $A$ site increases linearly as $y$ increases, which can be confirmed from the $y$ dependence of the magnetization. We plot the $y$ dependence of the saturated magnetization $\left(M_{\text {sat }}\right)$ along the $c$ axis in Fig. 5(d). $M_{\text {sat }}$ shows good coincidence with $y$-linear fitting, which is predicted by the formula

$$
M_{\text {sat }}=-(1-2 y) M_{A}+M_{B},
$$

where $M_{A}$ and $M_{B}$ are the saturated magnetization for $A$ and $B$ sublattices, respectively [34]. Here, we assume the selective $A$-site substitution with $\mathrm{Zn}$. This assumption is supported by the well-known chemical preference of $\mathrm{Zn}^{2+}$ in the tetrahedral coordination ( $A$ site) rather than in the octahedral coordination ( $B$ site), and also by the results of Mössbauer spectroscopy by Varret et al. [19] done on the same solid solution system. In the following, we discuss the origin of the $\mathrm{Zn}$-induced sign change of $\alpha$ via the site occupancy of $\mathrm{Zn}$ for the $A$ site.

\section{DISCUSSION}

In the present system, we observe two types of $\mathrm{ME}$ responses, i.e., the $H$-induced jump of $P$ due to the metamagnetic transition and the linear ME effect in the ferrimagnetic phase. We first briefly discuss the origin of the former. From Figs. 2(b) and 2(d), the magnetic-orderinduced electric polarizations $P_{\text {mag }}$ in the antiferromagnetic and the ferrimagnetic phases are approximately $P_{\mathrm{AF}}=$ $-1400 \mu \mathrm{C} / \mathrm{m}^{2}$ and $P_{\mathrm{FM}}=-800 \mu \mathrm{C} / \mathrm{m}^{2}$, respectively. Among several known mechanisms for spin-induced $P$ such as the exchange-striction mechanism [35], the spindependent metal-ligand $(d-p)$ hybridization mechanism [36], and the inverse Dzyaloshinskii-Moriya (D-M) mechanism $[37,38]$, the first two contribute to both $P_{\mathrm{AF}}$ and $P_{\mathrm{FM}}$, and the last one is irrelevant because it requires mutually canted neighboring spins. As for the origin of the jump of $P$, the fact that the two magnetic phases show different
$\left|P_{\text {mag }}\right|$ supports the exchange-striction scenario, where the lattice deforms to gain the exchange energy [39], while the $d-p$ hybridization mechanism is irrelevant because it is independent of the direction of spins with respect to the $c$ axis.

Next, we focus on the sign change of $\alpha$ by $\mathrm{Zn}$ doping [Fig. 5(d)]. Before entering in a discussion on the microscopic origin of the linear ME effect, we note that the $\mathrm{Zn}$-induced sign change of $\alpha$ can be explained by a simple model with competing ME mechanisms. We assume that the total ME coefficient $\alpha$ is the summation of two ME coefficients $\alpha_{A}$ and $\alpha_{B}$ originating from single-site ME mechanisms for the $A$ and $B$ sites, respectively. In this case, the magnitude of $\alpha_{A}$ is proportional to $1-2 y$, as Fe for the $A$ site selectively substituted by the doped $\mathrm{Zn} . \alpha$ is expressed as follows:

$$
\alpha=(1-2 y) \alpha_{A}+\alpha_{B},
$$

which gives the $y$-linear dependence of $\alpha$ as observed for $\alpha_{1}$ and $\alpha_{3}$. When $\alpha_{A}<0$ and $\alpha_{B}>0$ and $\left|\alpha_{A}\right|>\alpha_{B}$ are satisfied, the sign change of $\alpha$ is thus clearly a competition between $\alpha_{A}$ and $\alpha_{B}$; for low $y(\sim 0)$, the system is dominated by $\alpha_{A}$ with a negative value, while for high $y(\sim 0.5)$, the $\alpha_{B}$ contribution becomes dominant, leading to the sign change of $\alpha$. As for the linear ME effect, several mechanisms have been proposed [3]: the $g$-factor mechanism and the singlesite-anisotropy mechanism, originating from individual magnetic sites, as well as the exchange-striction mechanism and the inverse D-M mechanism for the two-site mechanisms, which are related to the correlation between neighboring spins. For $\alpha_{3}$, at the lowest $T(2 \mathrm{~K})$, only the $g$-factor mechanism [40] is relevant, which induces $P$ because of a shift of the $\mathrm{Fe}$ ion or surrounding $\mathrm{O}$ ions to gain the Zeeman energy or increase the $g$ factor of the single magnetic site. Following the above discussion, the $g$-factor mechanisms for respective $A$ and $B$ sites correspond to the single-site ME coefficients $\alpha_{A}$ and $\alpha_{B}$. From the linear fitting of $\alpha_{3}$ in Fig. 5(d), we obtain $\alpha_{A}=-43 \mathrm{ps} / \mathrm{m}$ and $\alpha_{B}=23 \mathrm{ps} / \mathrm{m}$. In addition to the large negative ME coefficient for the $A$ sites, the $B$ sites still show a large linear ME signal, leading to the wide-range variation of $\alpha_{3}$ from a large negative to a large positive value.

As for $\alpha_{1}$, both the single-site-anisotropy mechanism [41] and the $g$-factor mechanism can be relevant, while our current study cannot eliminate the contribution from the inverse D-M mechanism [42] for the correlation between spins at $A$ and $B$ sites because the magnitude of this mechanism is also proportional to $1-2 y$, the occupancy of Fe for the $A$ site. Nevertheless, it is possible that multiple mechanisms are competing in this system. For $y=0.5$, only the single-site mechanisms for the $B$ site are active, the total value of which is $+98 \mathrm{ps} / \mathrm{m}$. The sign change of $\alpha_{1}$ as $y$ decreases, like $\alpha_{3}$, strongly suggests that the other mechanisms such as the single-site mechanisms for the 
$A$ site and/or the inverse D-M mechanism produce several times larger ME signals with a negative sign for $y=0.125$ and 0.25 . Further studies are desired to identify the microscopic mechanism and explain the large magnitude of $\alpha$.

In conclusion, we showed the strong ME coupling in a polar antiferromagnet $\mathrm{Fe}_{2} \mathrm{Mo}_{3} \mathrm{O}_{8}$ and the $\mathrm{Zn}$-doping tunability of its magnetic and ME properties. In this system, two different phases, antiferromagnetic and ferrimagnetic phases, are competing with each other, and the balance between them is controlled by both the magnetic field and $\mathrm{Zn}$ doping. Increasing the doped amount of $\mathrm{Zn}$ stabilizes the ferrimagnetic order endowed with a large linear ME effect. The ME coefficient linearly changes with Zn-doping content and shows the sign change; by doping $\mathrm{Zn}$ atoms from $12.5 \%$ to $50 \%, \alpha_{3}\left(\Delta P_{c}=\alpha_{3} H_{c}\right)$ varies from $-11 \mathrm{ps} / \mathrm{m}$ to $21 \mathrm{ps} / \mathrm{m}$ and $\alpha_{1}\left(\Delta P_{b^{\prime}}=\alpha_{1} H_{b^{\prime}}\right)$ varies from $-142 \mathrm{ps} / \mathrm{m}$ to $107 \mathrm{ps} / \mathrm{m}$. We suggest that such a remarkable tunability of the linear ME coefficients originates from the presence of plural competing ME mechanisms and the selective control of them. This becomes possible because of the chemical preference of $\mathrm{Zn}$ ions for one of two distinct magnetic sites in the unit cell. The present work exemplifies the potential of chemical doping to achieve high functionality in ME material with spin frustration.

\section{ACKNOWLEDGMENTS}

The authors thank N. Nagaosa, T. Arima, Y. Motome, T. Ideue, and L. Ye for enlightening discussions. This work was partly supported by Grants-In-Aid for Scientific Research (Grant No. 24224009) from the MEXT of Japan and the FIRST Program by JSPS.

[1] I. E. Dzyaloshinskii, On the Magneto-Electrical Effect in Antiferromagnets, Zh. Eksp. Theor. Fiz. 37, 881 (1959) [Sov. Phys. JETP 10, 628 (1960)].

[2] D. N. Astrov, The Magnetoelectric Effect in Antiferromagnetics, Sov. Phys. JETP 11, 708 (1960).

[3] A. J. Freeman and H. Schmid, Magnetoelectric Interaction Phenomena in Crystals (Gordon and Breach Science Publishers, London, 1975).

[4] H. Schmid, Introduction to the Proceedings of the 2nd International Conference on Magnetoelectric Interaction Phenomena in Crystals, MEIPIC-2, Ferroelectrics 161, 1 (1994).

[5] J. F. Scott, Applications of Modern Ferroelectrics, Science 315, 954 (2007).

[6] M. Fiebig, Revival of the Magnetoelectric Effect, J. Phys. D 38, R123 (2005).

[7] C. A. F. Vaz, Electric Field Control of Magnetism in Multiferroic Heterostructures, J. Phys. Condens. Matter 24, 333201 (2012).

[8] S. W. Cheong and M. Mostovoy, Multiferroics: A Magnetic Twist for Ferroelectricity, Nat. Mater. 6, 13 (2007).
[9] W. Eerenstein, N. D. Mathur, and J. F. Scott, Multiferroicity and Magnetoelectric Materials, Nature (London) 442, 759 (2006).

[10] T. Kimura, T. Goto, H. Shintani, K. Ishizaka, T. Arima, and Y. Tokura, Magnetic Control of Ferroelectric Polarization, Nature (London) 426, 55 (2003).

[11] J. Wang, J. B. Neaton, H. Zheng, V. Nagarajan, S. B. Ogale, B. Liu, D. Viehland, V. Vaithyanathan, D. G. Schlom, U. V. Waghmare, N. A. Spaldin, K. M. Rabe, M. Wuttig, and R. Ramesh, Epitaxial $\mathrm{BiFeO}_{3}$ Multiferroic Thin Film Heterostructures, Science 299, 1719 (2003).

[12] D. A. Sanchez, N. Ortega, A. Kumar, R. Roque-Malherbe, R. Polanco, J. F. Scott, and R.S. Katiyar, Symmetries and Multiferroic Properties of Novel Room-Temperature Magnetoelectrics: Lead Iron Tantalate-Lead Zirconate Titanate (PFT/PZT), AIP Adv. 1, 042169 (2011).

[13] S. Seki, Y. Yamasaki, Y. Shiomi, S. Iguchi, Y. Onose, and Y. Tokura, Impurity-Doping-Induced Ferroelectricity in the Frustrated Antiferromagnet $\mathrm{CuFeO}_{2}$, Phys. Rev. B 75, 100403 (2007).

[14] R. P. Chaudhury, F. Ye, J. A. Fernandez-Baca, B. Lorenz, Y. Q. Wang, Y. Y. Sun, H. A. Mook, and C. W. Chu, Robust Ferroelectric State in Multiferroic $\mathrm{Mn}_{1-x} \mathrm{Zn}_{x} \mathrm{WO}_{4}$, Phys. Rev. B 83, 014401 (2011).

[15] W. H. McCarroll, L. Katz, and R. Ward, Some Ternary Oxides of Tetravalent Molybdenum, J. Am. Chem. Soc. 79, 5410 (1957).

[16] F. A. Cotton, Metal Atom Clusters in Oxide Systems, Inorg. Chem. 3, 1217 (1964).

[17] S. P. McAlister and P. Strobel, Magnetic Order in $\mathrm{M}_{2} \mathrm{Mo}_{3} \mathrm{O}_{8}$ Single Crystals $(M=\mathrm{Mn}, \mathrm{Fe}, \mathrm{Co}, \mathrm{Ni})$, J. Magn. Magn. Mater. 30, 340 (1983).

[18] D. Bertrand and H. Kerner-Czeskleba, Étude Structurale et Magnétique de Molybdates d'éléments de Transition, J. Phys. 36, 379 (1975).

[19] F. Varret, H. Czeskleba, F. Hartmann-Boutron, and P. Imbert, Étude par effet Mössbauer de l'ion $\mathrm{Fe}^{2+}$ en Symétrie Trigonale Dans les Composés du Type $(\mathrm{Fe}, M)_{2} \mathrm{Mo}_{3} \mathrm{O}_{8}$ ( $M=\mathrm{Mg}, \mathrm{Zn}, \mathrm{Mn}$, Co, Ni) et Propriétés Magnétiques de $(\mathrm{Fe}, \mathrm{Zn})_{2} \mathrm{Mo}_{3} \mathrm{O}_{8}$, J. Phys. 33, 549 (1972).

[20] Y. Tokura, Critical Features of Colossal Magnetoresistive Manganites, Rep. Prog. Phys. 69, 797 (2006).

[21] P. Strobel and Y. Le Page, Growth and Morphology of Single Crystals of Hexagonal Molybdates(IV) $\mathrm{M}_{2} \mathrm{Mo}_{3} \mathrm{O}_{8}$ ( $M=\mathrm{Mn}, \mathrm{Fe}, \mathrm{Co}, \mathrm{Ni}), \mathrm{J}$. Cryst. Growth 61, 329 (1983).

[22] P. Strobel, Y. Le Page, and S. P. McAlister, Growth and Physical Properties of Single Crystals of $\mathrm{Fe}_{2} \mathrm{Mo}_{3} \mathrm{O}_{8}$, J. Solid State Chem. 42, 242 (1982).

[23] R. E. Newnham, Properties of Materials: Anisotropy, Symmetry, Structure (Oxford, England, 1959).

[24] S. Nakayama, R. Nakamura, M. Akaki, D. Akahoshi, and H. Kuwahara, Ferromagnetic Behavior of $\left(\mathrm{Fe}_{1-y} \mathrm{Zn}_{y}\right)_{2} \mathrm{Mo}_{3} \mathrm{O}_{8}$ $(0 \leq y \leq 1)$ Induced by Nonmagnetic Zn Substitution, J. Phys. Soc. Jpn. 80, 104706 (2011).

[25] We confirmed that the ferrimagnetic phase is retained even in the zero field at $10 \mathrm{~K}$ and $5 \mathrm{~K}$ for $y=0.05$ (not shown) in the $H$ decreasing scan of $M$ after field cooling with $\mu_{0} H=5 \mathrm{~T}$.

[26] E. Ascher, H. Rieder, H. Schmid, and H. Stossel, Some Properties of Ferromagnetoelectric Nickel-Iodine Boracite, $\mathrm{Ni}_{3} \mathrm{~B}_{7} \mathrm{O}_{13}$ I, J. Appl. Phys. 37, 1404 (1966). 
[27] T. Arima, D. Higashiyama, Y. Kaneko, J. P. He, T. Goto, S. Miyasaka, T. Kimura, K. Oikawa, T. Kamiyama, R. Kumai, and Y. Tokura, Structural and Magnetoelectric Properties of $\mathrm{Ga}_{2-x} \mathrm{Fe}_{x} \mathrm{O}_{3}$ Single Crystals Grown by a Floating-Zone Method, Phys. Rev. B 70, 064426 (2004).

[28] G. T. Rado, Observation and Possible Mechanisms of Magnetoelectric Effects in a Ferromagnet, Phys. Rev. Lett. 13, 335 (1964).

[29] G. T. Rado and J. M. Ferrari, Linear and Bilinear Magnetoelectric Effects in Magnetically Biased Magnetite $\left(\mathrm{Fe}_{3} \mathrm{O}_{4}\right)$, Phys. Rev. B 15, 290 (1977).

[30] K. Siratori, E. Kita, G. Kaji, A. Tasaki, S. Kimura, I. Shindo, and K. Kohn, Magnetoelectric Effect of $\mathrm{Fe}_{3} \mathrm{O}_{4}$ at 77 K. I. Crystal Symmetry, J. Phys. Soc. Jpn. 47, 1779 (1979).

[31] K. Siratori and E. Kita, Jiseitai no Denki-jiki Koka, Kotai Butsuri (AGNE Gijutsu Center, Tokyo, Japan) 14, 599 (1979).

[32] G. T. Rado, J. M. Ferrari, and W. G. Maisch, Magnetoelectric Susceptibility and Magnetic Symmetry of Magnetoelectrically Annealed $\mathrm{TbPO}_{4}$, Phys. Rev. B 29, 4041 (1984).

[33] R. O. Cherifi, V. Ivanovskaya, L. C. Phillips, A. Zobelli, I. C. Infante, E. Jacquet, V. Gercia, S. Fusil, P. R. Briddon, N. Guiblin, A. Mougin, A. A. Unal, F. Kronast, S. Valencia, B. Dkhil, A. Barthelemy, and M. Bibes, Electric-Field Control of Magnetic Order above Room Temperature, Nat. Mater. 13, 345 (2014).

[34] Previous studies [18,19] have revealed that $M_{B}$ is larger than $M_{A}$, which makes $M_{B}\left(M_{A}\right)$ parallel (antiparallel) to the applied $H$ along the $c$ axis.
[35] I. A. Sergienko, C. Sen, and E. Dagotto, Ferroelectricity in the Magnetic E-Phase of Orthorhombic Perovskites, Phys. Rev. Lett. 97, 227204 (2006).

[36] T. Arima, Ferroelectricity Induced by Proper-Screw Type Magnetic Order, J. Phys. Soc. Jpn. 76, 073702 (2007).

[37] H. Katsura, N. Nagaosa, and A. V. Balatsky, Spin Current and Magnetoelectric Effect in Noncollinear Magnets, Phys. Rev. Lett. 95, 057205 (2005).

[38] I. A. Sergienko and E. Dagotto, Role of DzyaloshinskiiMoriya Interaction in Multiferroic Perovskites, Phys. Rev. B 73, 094434 (2006).

[39] The exchange energy is proportional to $\boldsymbol{S}_{i} \cdot \boldsymbol{S}_{j}$, where $\boldsymbol{S}_{i}$ is the spin operator for the $i$ th site. In the antiferromagnetic phase, the interlayer couplings [bond 2 in Fig. 1(b)] are ferromagnetic $\left(\boldsymbol{S}_{i} \cdot \boldsymbol{S}_{j}>0\right)$, while the nearest-neighbor coupling (bond 1) is antiferromagnetic. In the ferrimagnetic phase, on the other hand, bond 2 flops to antiferromagnetic $\left(\boldsymbol{S}_{i} \cdot \boldsymbol{S}_{j}<0\right)$ while bond 1 remains antiferromagnetic. Such a feature should lead to the difference in magnitude of exchange striction between the antiferromagnetic and the ferrimagnetic phases, resulting in the difference of $P_{\mathrm{mag}}$.

[40] S. Alexander and S. Shtrikman, On the Origin of the Axial Magnetoelectric Effect of $\mathrm{Cr}_{2} \mathrm{O}_{3}$, Solid Stat. Commn. 4, 115 (1966).

[41] G. T. Rado, Mechanism of the Magnetoelectric Effect in an Antiferromagnet, Phys. Rev. Lett. 6, 609 (1961).

[42] R. Hornreich and S. Shtrikman, Statistical Mechanics and Origin of the Magnetoelectric Effect in $\mathrm{Cr}_{2} \mathrm{O}_{3}$, Phys. Rev. 161, 506 (1967). 